\title{
Surgical clipping or endovascular coiling for unruptured intracranial aneurysms: a pragmatic randomised trial
}

\author{
Tim E Darsaut, ${ }_{1}^{1}$ J Max Findlay, ${ }^{1}$ Elsa Magro, ${ }^{2}$ Marc Kotowski, ${ }^{3}$ Daniel Roy, ${ }^{3}$ \\ Alain Weill, ${ }^{3}$ Michel W Bojanowski, ${ }^{4}$ Chiraz Chaalala, ${ }^{4}$ Daniela lancu, ${ }^{5}$ Howard Lesiuk, \\ John Sinclair, ${ }^{6}$ Felix Scholtes, ${ }^{7}$ Didier Martin, ${ }^{7}$ Michael M Chow, ${ }^{1}$ Cian J O'Kelly, ${ }_{1}$ \\ John H Wong, ${ }^{8}$ Ken Butcher, ${ }^{9}$ Allan J Fox ${ }^{10}$ Adam S Arthur, ${ }^{11}$ Francois Guilbert, ${ }^{3}$ \\ Lu Tian, ${ }^{12}$ Miguel Chagnon, ${ }^{13}$ Suzanne Nolet, ${ }^{14}$ Guylaine Gevry, ${ }_{1}^{14}$ Jean Raymond ${ }^{3}$
}

- Additional material is published online only. To view please visit the journal online (http://dx.doi.org/10.1136/ jnnp-2016-315433)

For numbered affiliations see end of article.

\section{Correspondence to} Dr Jean Raymond, CHUM Notre-Dame Hospital, Department of Radiology 1560, Sherbrooke East, Pavilion Simard, Suite Z12909, Montreal, Quebec H2L 4M1, Canada; jean. raymond@umontreal.ca

Received 7 December 2016 Revised 30 March 2017 Accepted 13 April 2017 Published Online First 20 June 2017
CrossMark

\section{To cite: Darsaut TE,} Findlay JM, Magro E, et al. J Neurol Neurosurg Psychiatry 2017:88:663-668.

\section{ABSTRACT}

Background Unruptured intracranial aneurysms (UIAs) are increasingly diagnosed and are commonly treated using endovascular treatment or microsurgical clipping. The safety and efficacy of treatments have not been compared in a randomised trial. How to treat patients with UIAs suitable for both options remains unknown. Methods We randomly allocated clipping or coiling to patients with one or more 3-25 mm UIAs judged treatable both ways. The primary outcome was treatment failure, defined as: initial failure of aneurysm treatment, intracranial haemorrhage or residual aneurysm on 1-year imaging. Secondary outcomes included neurological deficits following treatment, hospitalisation $>5$ days, overall morbidity and mortality and angiographic results at 1 year.

Results The trial was designed to include 260 patients. An analysis was performed for slow accrual: 136 patients were enrolled from 2010 through 2016 and 134 patients were treated. The 1-year primary outcome, available for 104 patients, was reached in 5/48 (10.4\% $(4.5 \%-22.2 \%))$ patients allocated surgical clipping, and 10/56 (17.9\% (10.0\%-29.8\%)) patients allocated endovascular coiling (OR: $0.54(0.13-1.90), p=0.40)$. Morbidity and mortality (modified Rankin Scale $>2$ ) at 1 year occurred in $2 / 48(4.2 \%(1.2 \%-14.0 \%))$ and 2/56 (3.6\% (1.0\%-12.1\%)) patients allocated clipping and coiling, respectively. New neurological deficits (15/65 vs 6/69; OR: 3.12 (1.05-10.57), $p=0.031)$, and hospitalisations beyond 5 days (30/65 vs 6/69; OR: 8.85 (3.22-28.59), $p=0.0001$ ) were more frequent after clipping.

Conclusion Surgical clipping or endovascular coiling of UIAs did not show differences in morbidity at 1 year. Trial continuation and additional randomised evidence will be necessary to establish the supposed superior efficacy of clipping.

\section{INTRODUCTION}

Unruptured intracranial aneurysms (UIAs) are relatively common, with an estimated prevalence of $2 \%-5 \%{ }^{1}$ They are increasingly diagnosed due to the widespread availability of non-invasive imaging. ${ }^{12}$ An increasing number of patients are undergoing aneurysm repair, particularly with coiling, mainly to prevent the often devastating effects of subarachnoid haemorrhage (SAH), but the benefits of any preventive treatment have never been proven. ${ }^{3-5}$

There are two ways to repair aneurysms. Surgical clipping is gradually being replaced by less invasive endovascular treatment. ${ }^{16}$ For ruptured aneurysms, this shift in practice is supported by the results of the International Subarachnoid Aneurysm Trial (ISAT) that demonstrated improved 1-year clinical outcomes with coiling as compared with clipping. ${ }^{7}$ However, the results for ruptured aneurysms do not apply to UIAs: the added morbidity of surgery seen in ISAT cannot be extrapolated to surgery for UIAs, as conditions for surgical treatment are more favourable in the elective, unruptured setting. Furthermore, the two settings differ in fundamental clinical aims. In the situation of a ruptured aneurysm, the primary goal of treatment is to ensure that the patient survives the acute phase of SAH with minimal morbidity and without aneurysm rebleeding. In this context, the presence of residual or development of recurrent aneurysm, generally conceded to be more common with aneurysm coiling, is less of a concern than with UIA, where the primary goal is lifelong protection from aneurysm rupture. ${ }^{89}$ Here, the problem of residual or recurrent aneurysm may require surveillance imaging, or even warrant retreatment with additional risks. ${ }^{10-12}$

Proponents of surgery believe that it has superior efficacy and durability compared with coiling. Endovascular repair, on the other hand, has the appeal of a less invasive procedure and perhaps decreased treatment-related morbidity. Neither of these claims been demonstrated in a randomised trial. $^{12-19}$ The best available comparative evidence are the results of meta-analyses of observation studies of outcomes following surgical clipping ${ }^{14}$ or endovascular coiling, ${ }^{16}$ a large international registry $^{20}$ and National Inpatient Sample (NIS) database data ${ }^{1-23}$ but these are subject to the limitations of observational studies. ${ }^{24}$

There exists significant variability and disagreement among clinicians on how best to manage patients with UIA suitable for repair with either approach. ${ }^{25}$

We reasoned that these patients would be best managed within the context of a pragmatic trial, with 


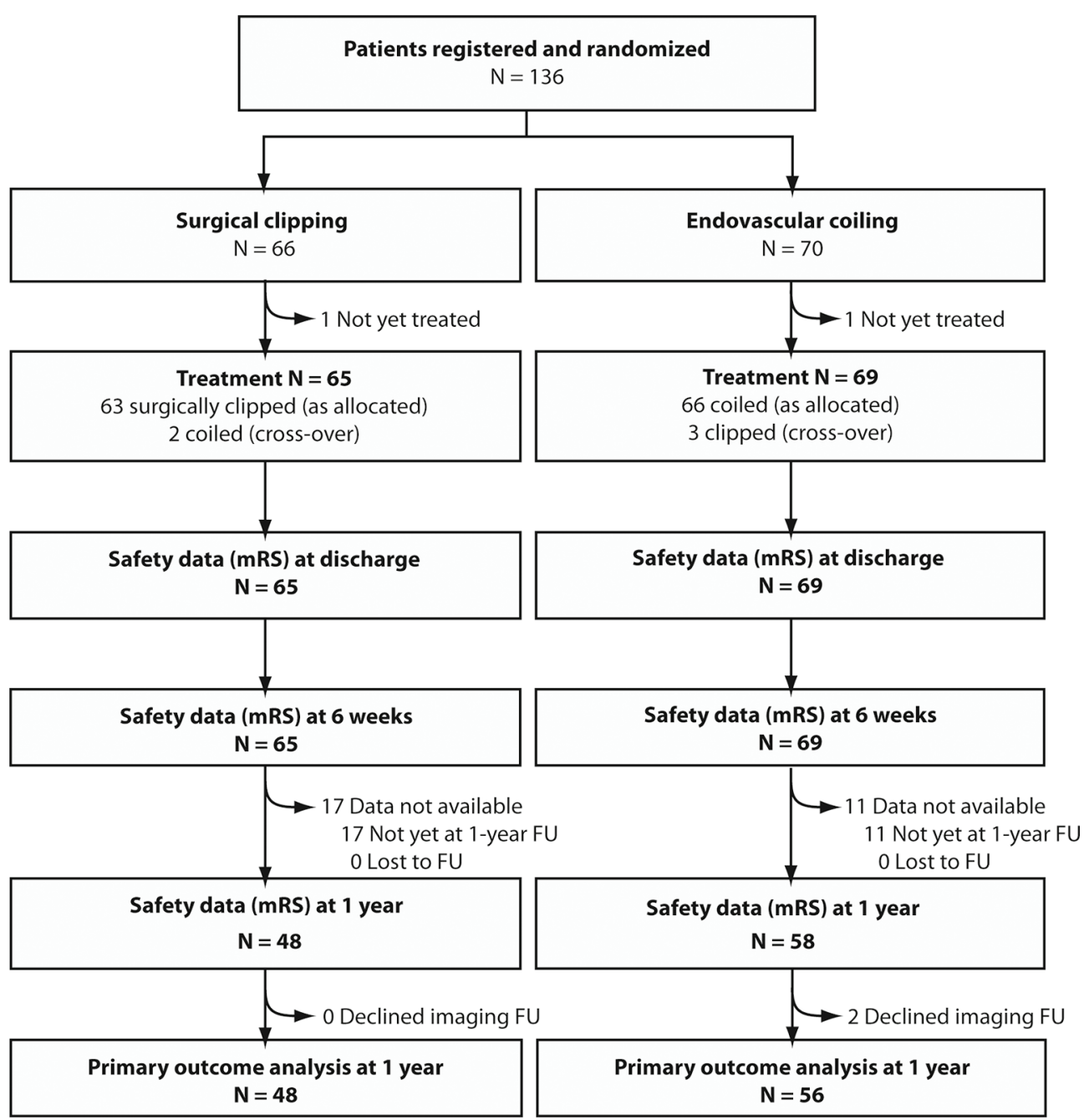

Figure 1 Flowchart. mRS, modified Rankin Scale.

the randomised allocation offering an equal chance of getting the best treatment, which for the time being remains unknown. The Collaborative UnRuptured Endovascular versus Surgery (CURES) study was launched in 2010 to examine trial feasibility and to compare angiographic and clinical outcomes following clipping and coiling after 1 year.

\section{METHODS}

CURES is an investigator-led, pragmatic, multicentre randomised $(1: 1)$ parallel-group trial conducted in four Canadian and one European centres. The hypothesis was that 'Surgical clipping of intradural, saccular, UIAs is superior to endovascular management in terms of decreasing the number of patients experiencing treatment failure from $14 \%$ to $3 \%{ }^{26}$ The number of patients needed to demonstrate this difference was estimated to be 118 per group (with a statistical power of 0.80 and a two-sided type 1 error of 0.05 ), or 260 patients (to account for loss to follow-up and cross-overs). Blinded data were examined by the Data Safety Monitoring Committee (DSMC) at preplanned 100 patient intervals, but there were no prespecified stopping rules. Data capture and management were through secure servers in compliance with Good Clinical Practice requirements. The protocol was approved by all local Institutional Review Boards, and all patients provided written informed consent prior to participation. Case report forms were simple, and data collected parsimonious, in order to facilitate completion by normal care personnel. Screening logs were not required by protocol.

\section{Patients}

The trial was designed to address the question of the best treatment for patients with UIAs eligible for both endovascular and surgical repair. Independent (modified Rankin Scale (mRS) score of $\leq 2$ ) patients 18 years and older with any intradural saccular UIAs $3-25 \mathrm{~mm}$ in maximal cross-sectional diameter were offered participation if they had at least 10 years of life expectancy. Aneurysms were excluded if they were thought to require endovascular flow diversion or parent vessel occlusion, with or without a bypass. Patients with multiple aneurysms were not excluded. The protocol was not modified other than to include, starting in October 2014, patients with recurrent previously treated aneurysms (two patients). The trial involved five tertiary neurosurgical centres that offer both clipping and coiling (in Canada: Calgary, Edmonton, Montreal and Ottawa; in Belgium: Liège).

\section{Interventions}

Patients were treated with surgical clipping or endovascular treatment as per local practices, with technical details regarding treatment left to the individual operators. 
Table 1 Patient and aneurysm characteristics

\begin{tabular}{|c|c|c|}
\hline & Surgical & Endovascular \\
\hline Characteristic & $n=66$ & $\mathrm{n}=70$ \\
\hline \multicolumn{3}{|l|}{ Patient } \\
\hline Mean age at treatment, year (SD) & $56(8)$ & $57(7)$ \\
\hline Female sex, n (\%) & $41(62 \%)$ & $53(76 \%)$ \\
\hline Pretreatment mRS score $=0, \mathrm{n}(\%)$ & $55(83 \%)$ & $47(67 \%)$ \\
\hline$=1, \mathrm{n}(\%)$ & $10(15 \%)$ & $19(27 \%)$ \\
\hline$=2, n(\%)$ & 0 & $3(4 \%)$ \\
\hline No mRS: patients not yet treated & $1(2 \%)$ & $1(2 \%)$ \\
\hline Patients with multiple aneurysms & 12 & 18 \\
\hline \multicolumn{3}{|l|}{ Index aneurysm } \\
\hline \multicolumn{3}{|l|}{ Location } \\
\hline Anterior circulation & 65 & 66 \\
\hline Ophthalmic/paraophthalmic & 9 & 8 \\
\hline Posterior communicating & 7 & 12 \\
\hline Carotid terminus & 6 & 8 \\
\hline MCA bifurcation & 18 & 15 \\
\hline Anterior communicating & 19 & 13 \\
\hline Other anterior circulation location & 6 & 10 \\
\hline Posterior circulation & 1 & 4 \\
\hline Index aneurysm size (mm), mean (range) & $8.7(3-20)$ & $8.2(3-23)$ \\
\hline Aneurysm size $3-10 \mathrm{~mm}$ & 53 & 53 \\
\hline Size $11-15 \mathrm{~mm}$ & 8 & 12 \\
\hline Size $16-24 \mathrm{~mm}$ & 4 & 3 \\
\hline Recurrent, previously treated index aneurysm & 1 & 1 \\
\hline Symptomatic aneurysms & 12 & 10 \\
\hline Pain/headache & 6 & 10 \\
\hline Cranial nerve palsy & 2 & 0 \\
\hline Stroke/TIA & 4 & 0 \\
\hline $\begin{array}{l}\text { History of previous SAH from another } \\
\text { aneurysm }\end{array}$ & 7 & 7 \\
\hline Hypertension & 34 & 31 \\
\hline Current smoker & 26 & 30 \\
\hline Excessive alcohol & 7 & 3 \\
\hline Positive family history & 10 & 13 \\
\hline \multicolumn{3}{|l|}{ Treatment } \\
\hline $\begin{array}{l}\text { Time from randomisation to treatment, mean } \\
\text { weeks (SD) }\end{array}$ & $14(6)$ & $6(1)$ \\
\hline Adherence to assigned treatment, $\mathrm{n}(\%)$ & $63 / 65(97 \%)$ & $66 / 69(96 \%)$ \\
\hline $\begin{array}{l}\text { Index aneurysm rupture after randomisation, } \\
\text { prior to treatment }\end{array}$ & 1 & 0 \\
\hline $\begin{array}{l}\text { Additional non-index aneurysms treated at } \\
\text { same time }\end{array}$ & 12 & 5 \\
\hline
\end{tabular}

MCA, Middle Cerebral Artery; mRS, modified Rankin Scale; SAH, subarachnoid haemorrhage; TIA, transient ischemic attack.

\section{Outcome measures}

The primary outcome measure of treatment failure was considered to have occurred at 1 year with (1) initial failure of aneurysm treatment using the allocated modality; or (2) intracranial haemorrhage during follow-up; or (3) a residual or recurrent aneurysm found using Computed Tomographic Angiography (CTA), Magnetic Resonance Angiography (MRA) or Digital Subtraction Angiography(DSA) during follow-up. Cross-overs to the other treatment arm with no attempt to occlude the aneurysm using the randomly allocated modality were not considered treatment failures.

Other endpoints included overall morbidity $(m R S>2)$ and mortality at 1 year, new perioperative (30 days) neurological deficits (defined as any new weakness, sensory abnormality, decreased level of consciousness or cranial nerve deficit),

\begin{tabular}{|c|c|c|c|}
\hline Intent-to-treat analysis & Surgical & Endovascular & \\
\hline 1-year outcome & $n=48$ & $n=56$ & OR \\
\hline Treatment failure (composite) & 5 & 10 & $\begin{array}{l}0.54(0.13,1.90) \\
p=0.40\end{array}$ \\
\hline $\begin{array}{l}\text { Failure to treat aneurysm with } \\
\text { allocated modality }\end{array}$ & 1 & 3 & $\begin{array}{l}0.38(0.01,4.91), \\
p=0.62\end{array}$ \\
\hline $\begin{array}{l}\text { Intracranial haemorrhage } \\
\text { during first-year FU }\end{array}$ & 1 & 1 & $\begin{array}{l}1.17(0.01,93.44) \\
p=1.00\end{array}$ \\
\hline Saccular residual aneurysm & 3 & 6 & $\begin{array}{l}0.56(0.09,2.80) \\
p=0.51\end{array}$ \\
\hline
\end{tabular}

\begin{tabular}{lll}
\hline As-treated analysis & & \\
\hline 1-year outcome & $\mathrm{n}=49$ & $\mathrm{n}=55$ \\
\hline Treatment failure (composite) & 5 & 10 \\
$\begin{array}{l}\text { Failure to treat aneurysm with } \\
\text { allocated modality }\end{array}$ & 1 & 3 \\
$\begin{array}{l}\text { Intracranial haemorrhage } \\
\text { during first-year FU }\end{array}$ & 1 & 1 \\
Saccular residual aneurysm & 3 & 6 \\
\hline FU, Follow-Up. &
\end{tabular}

perioperative morbidity $(\mathrm{mRS}>2)$ measured at discharge, peritreatment hospitalisation lasting $>5$ days and discharge from hospital to a location other than home.

Follow-up tests and visits were standard per local practices, including neurological examinations, brain imaging studies and a functional assessment according to the mRS at discharge, 6 weeks and 1 year. A vascular imaging study at $12 \pm 2$ months to verify aneurysm occlusion was expected as standard care, with results centrally adjudicated by an independent diagnostic neuroradiologist (FG). The core lab classified angiographic results at 1 year according to a three-tier system (complete occlusion, residual neck, residual aneurysm) as reported in reference. ${ }^{10}$

\section{Randomisation}

Parallel-group randomisation (1:1) was concealed, generated through a web-based platform (MedSciNet). Minimisation criteria used were as follows: age $>60$, aneurysm size $\geq 15 \mathrm{~mm}$ and posterior circulation location. Posterior communicating artery aneurysms were considered to be in the anterior circulation. Randomisation and minimisation functions were tested to ensure that the processes were robust.

\section{Blinding}

In this pragmatic trial comparing open surgical and endovascular approaches, blinding to treatment assignment of patients, physicians and outcome assessors was not possible.

\section{Statistical analyses}

Analyses were performed by a statistician (LT) according to protocol. Baseline characteristics were compared between two groups using $\mathrm{t}$ tests or Fisher's exact tests, when appropriate. For primary and secondary endpoints, binomial proportions were estimated by treatment group and 95\% CIs constructed using the Wilson score method to account for small numbers. The primary outcome between the two treatment groups was compared using Fisher's exact test. The OR and associated 95\% $\mathrm{CI}$ were reported. The statistical significance level was set at 0.05 for a two-sided test. All statistical analyses were conducted using R.3.2.2 (R foundations for statistical computing). 


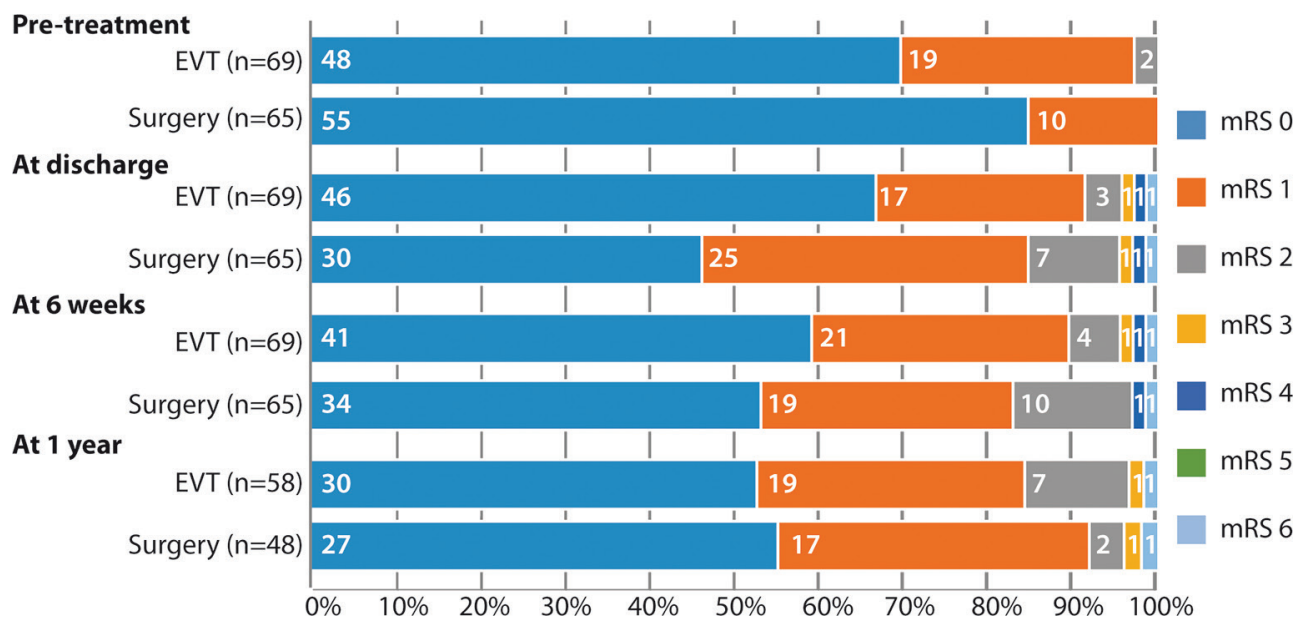

Figure 2 Functional outcome after endovascular coiling or after surgical clipping of unruptured intracranial aneurysm according to the modified Rankin Scale (mRS) score. EVT, Endovascular Treatment.

\section{Roles of the funding source and data integrity}

The study was funded by the CIHR (MOP 119554) and sponsored by the Centre Hospitalier de l'Université de Montréal. Neither funding agency nor sponsor had any part in the study design, data collection, analysis or reporting, and no access to the data or source documents. The full protocol was published ${ }^{26}$ and the latest version is available online (http://www.clinicalcare-trials.org/cures-study).

\section{RESULTS}

From September 2010 to May 2016, 136 patients with 181 UIAs (136 index aneurysms and 45 additional aneurysms) were recruited. On 9 May 2016, the Steering Committee (SC) submitted blinded 1-year outcome data on the first 100 patients to the DSMC. The DSMC had no concerns related to the conduct of the trial. Given the slow accrual, financial commitments to participants and deadlines for funding renewal, the SC decided to examine the trial results available to date.

The outcomes of all patients who were registered and randomised are presented in the trial profile (figure 1). The number of patients with UIAs managed outside the trial cannot be provided because eligibility logs were not required per protocol. Baseline patient and aneurysm characteristics (table 1) were similar, with no statistically significant differences. Of 136 patients, 63/66 patients that were randomly allocated clipping underwent clipping; two were coiled and one patient has not been treated. Of the 70 patients randomly allocated coiling, 66 underwent coiling, 3 patients were clipped and 1 has not been treated, leaving 134 patients for safety outcome analysis at 6 weeks. Details regarding treatments and complications are provided in online supplementary table 4 (see online supplementary tables).

The 1-year primary outcome data are available for 104/136 (76.5\%) patients (table 2). Ten of 56 patients $(17.9 \%$ (10.0\%$29.8 \%)$ in the endovascular group and 5 of $48(10.4 \%(4.5 \%-$ $22.2 \%)$ ) in the surgical group reached the primary outcome of 'treatment failure', defined as failure to treat the aneurysm, aneurysm remnant at 1 year or intracranial haemorrhage during follow-up. The OR of reaching the primary outcome for endovascular coiling versus surgical clipping was $0.54((0.13-1.90)$, $\mathrm{p}=0.40$ ). An as-treated analysis of the primary endpoint gave similar results. In the endovascular group, three patients could not be treated with coils, one had a fatal treatment-related SAH and six had saccular aneurysmal recurrences during follow-up (retreated by

\begin{tabular}{|c|c|c|c|}
\hline Clinical outcome & Surgical $n=65$ & Endovascular $\mathrm{n}=69$ & $\mathrm{p}$ Value \\
\hline Number of days hospitalised peritreatment, mean (median) (range) & $6.7(5)(2-25)$ & $3.9(1)(0-122)$ & $<0.001$ \\
\hline Postoperative morbidity (mRS>2) & $3 / 65(4.6 \%)$ & $3 / 69(4.3 \%)$ & 1.0 \\
\hline Patients with new neurological deficits following treatment & $16 / 65(24.6 \%)$ & $7 / 69(10.1 \%)$ & 0.038 \\
\hline \multicolumn{4}{|l|}{ Discharge location } \\
\hline Other hospital & $3(4.6 \%)$ & $1(1.4 \%)$ & \\
\hline Rehabilitation centre & $2(3.1 \%)$ & $2(2.9 \%)$ & \\
\hline Death & $1(1.5 \%)$ & $1(1.4 \%)$ & \\
\hline Morbidity and mortality at 1 year $(\mathrm{mRS}>2)$ & $2 / 48(4.2 \%)$ & $2 / 56(3.6 \%)$ & 1.0 \\
\hline Re-treatment of index aneurysm during follow-up & $1 / 65(1.5 \%)$ & $2 / 69(2.9 \%)$ & 1.0 \\
\hline
\end{tabular}


coiling (1), clipping (1) and left untreated (4)). In the surgical group, one clipped index aneurysm patient was immediately returned for clip removal when the patient awoke hemiparetic. The clip was left off the aneurysm. One surgical patient died following aneurysmal rupture the day prior to scheduled treatment and three other residual aneurysms were identified during follow-up (re-treated with stent-assisted coiling (1) and left untreated (2)). Three patients randomised to coiling crossed over to clipping, citing personal preference. Two patients crossed over from clipping to coiling, one citing personal preference, whereas the other randomised patient was crossed over by the surgical team, having changed their mind about eligibility for both treatments due to the presence of a calcified aneurysm neck. The five crossed-over patients all had successful aneurysm treatment.

Secondary outcomes, including $\mathrm{mRS}$ scores at time of discharge, 6 weeks and at 1 year of follow-up are presented in figure 2 and table 3 . New perioperative neurological deficits occurred more commonly in patients treated surgically $(16 / 65$ $(24.6 \% ; 15.8 \%-36.3 \%)$ vs $7 / 69(10.1 \% ; 5.0 \%-19.5 \%)$; OR: 2.87 (1.02-8.93), $\mathrm{p}=0.038$ ), as did hospitalisation for more than 5 days $(30 / 65(46.2 \% ; 34.6 \%-58.1 \%)$ vs $6 / 69(8.7 \%$; 4.0\%-17.7\%); OR: 8.85 (3.22-28.59), $\mathrm{p}=0.0001)$. Clinical follow-up to 1 year is available in 106 patients: $1 / 48$ (2.0\%; $(0.3 \%-10.9 \%))$ clipped versus $1 / 58(1.7 \%$; $(0.3 \%-9.1 \%))$ coiled patients had died, and 1/48 clipped versus $1 / 58$ coiled patients had become disabled (mRS $>2)$.

Two patients treated with coiling declined 1-year follow-up imaging. At 1 year, $45 / 48$ (93.8\%; 83.2\%-97.9\%) patients allocated surgical clipping and 50/56 (89.3\%; 78.5\%-95.0\%) allocated aneurysm coiling had no evidence of residual saccular aneurysm $(\mathrm{p}=0.49)$. Details regarding angiographic imaging outcomes are available in online supplementary table S5.

\section{DISCUSSION}

Surgical trials are difficult, particularly when they concern widely different but accepted and practiced therapies. ${ }^{27}$ Although clipping and coiling have been used to prevent the rupture of UIAs for decades, there is no direct evidence of clinical benefit from either treatment compared with simple observation of these lesions. This fundamental question was not addressed by this trial. A previous international attempt to compare outcomes of coiling and conservative management of UIAs was halted due to insufficient recruitment. ${ }^{4}$ We addressed the next most important problem concerning UIAs with a trial comparing the two most frequent active management strategies which we hoped would be more readily accepted by both candidate patients and treating physicians.

Because UIAs rupture infrequently, on the order of $1 \%$ per year, ${ }^{120}$ a proper comparison of preventive treatments would ideally require thousands of patients followed for 5-10 years, using a hard clinical outcome such as death and major morbidity as a primary endpoint. ${ }^{18}$ For practical reasons, we started with a smaller trial and a more modest objective, using a surrogate primary outcome, measured over a shorter 1-year time frame. The results of angiography are routinely used by clinicians to verify treatment efficacy in individual patients, and they are the most common choice of endpoint in trials of aneurysm therapy. ${ }^{28-31}$ Because coiling was reputed but not proven to be less morbid initially, and clipping was theoretically more durable, what was chosen was a hypothesis that could be verified within a feasibly short time frame, yet long enough for patients to recover from transient surgical treatment-related morbidity, or for coiled aneurysms to recur.
At 1 year, the hypothesised superior efficacy of clipping could not be shown, but the target number of patients was not reached and some coiled aneurysms are known to recur after 1 year. ${ }^{10}$

The trial does provide randomised evidence that surgical clipping is associated with longer hospitalisation and more frequent treatment-related neurological deficits, as suggested by epidemiological studies. ${ }^{13}{ }^{21-23} 32$ Many of these deficits were mild or transient, and did not lead to a difference in mRS scores at discharge. At 1 year, death and dependency remained similarly infrequent in both treatment groups.

Although less morbid a procedure in the short term, there is no guarantee that coiling provides better patient safety over the patient's lifetime. Microsurgical clipping could still prove to be better, if delayed rupture occurs more often in recurrent coiled aneurysms, or as repeated angiography and re-treatments with attendant complications are performed over time. ${ }^{11}$

Confronted with a slow-recruiting trial, there are two standard options: stop the trial and report inconclusive results, or continue the trial without reporting results for fear of endangering recruitment. This latter option makes sense if there is hope for the trial to eventually reach the target number of patients.

In this case, the SC considered neither option to be appropriate. The use of clipping is already declining, and accordingly so is microsurgical expertise in aneurysm repair. ${ }^{633}$ It would be unfortunate to prematurely abandon a surgical treatment that could provide better long-term clinical outcomes, if that is the case. In the mean time, many centres still offer clipping to some patients, but it will become increasingly difficult to justify a more invasive intervention if benefits cannot be demonstrated in real-world practice. Given the greater initial morbidity associated with clipping, the burden of the proof is on its practitioners to demonstrate that it is associated with long-term benefits. This can only be properly done in an Randomized Controlled Trial (RCT), which becomes a necessity if we are to provide outcome-based neurosurgical care.

The second option, continuing the trial, is thought to require concealment of ongoing results, which could be improperly used as justification for choosing one treatment over the other, rather than continue randomised allocation of management options. For UIAs, most clinicians already use non-randomised evidence and opinions to justify their choice of one type of management. ${ }^{134}$ Rather than draw premature conclusions for making clinical decisions outside the trial, the intent of transparent reporting is to reassure the neurovascular community and funding agencies that randomised trials can be implemented in practice, and that patients have acceptable outcomes at 1 year with both treatments. These data could also contribute to future meta-analyses. ${ }^{35}$

Although there are a small number of patients and centres, limiting the generalisability of the results, the patients in this trial had demographic and aneurysm characteristics similar to those reported in meta-analyses of UIA clipping and coiling outcomes. ${ }^{1416}$ Other limitations include the surrogate angiographic endpoint, lack of blinding and the short follow-up. Other aspects of this study are intrinsic to the pragmatic design choices, which in the spirit of care trials, placed patient interests first, and thus required no additional tests, risks, visits or costs. ${ }^{36}$

\section{CONCLUSION}

Surgical clipping or endovascular coiling of UIAs did not show differences in morbidity at 1 year. Trial continuation and additional randomised evidence will be necessary to establish the supposed superior efficacy of clipping. 


\section{Author affiliations}

'Department of Surgery, Division of Neurosurgery, University of Alberta, Edmonton, Canada

${ }^{2}$ Service de Neurochirurgie, CHU Cavale Blanche, INSERM UMR 1101 LaTIM, Brest, France

${ }^{3}$ Department of Radiology, Service of Neuroradiology, Centre Hospitalier de I'Université de Montréal (CHUM), Notre-Dame Hospital, Montreal, Canada ${ }^{4}$ Department of Surgery, Service of Neurosurgery, Centre Hospitalier de l'Université de Montréal (CHUM), Notre-Dame Hospital, Montreal, Canada

${ }^{5}$ Department of Medical Imaging, Section of Neuroradiology, University of Ottawa, The Ottawa Hospital, Ottawa, Canada

${ }^{6}$ Department of Surgery, Section of Neurosurgery, University of Ottawa, The Ottawa Hospital, Ottawa, Canada

${ }^{7}$ Department of Neurosurgery, Centre Hospitalier Universitaire de Liège, Liège, Belgium

${ }^{8}$ Division of Neurosurgery, Department of Clinical Neurosciences, Hotchkiss Brain Institute, University of Calgary, Alberta, Canada

${ }^{9}$ Department of Medicine, Division of Neurology, University of Alberta, Edmonton, Canada

${ }^{10}$ Department of Medical Imaging, Sunnybrook Health Sciences Centre, University of Toronto, Toronto, Canada

${ }^{11}$ Department of Neurosurgery, Semmes-Murphey Neurologic and Spine Institute, University of Tennessee, Memphis, USA

${ }^{12}$ Department of Biomedical Data Science, Stanford University School of Medicine, Stanford, California, USA

${ }^{13}$ Department of Mathematics and Statistics, Université de Montréal, Montreal, Canada

${ }^{14}$ Research Centre, Centre Hospitalier de I'Université de Montréal (CHUM), NotreDame Hospital, Montreal, Canada

Contributors TED, JR and JMF designed the study and wrote the report; MC designed the statistical analysis, which was performed by LT; TED, JR, JMF, EM, MK, $\mathrm{DR}, \mathrm{AW}, \mathrm{MWB}, \mathrm{CC}, \mathrm{DI}, \mathrm{HL}, \mathrm{JS}, \mathrm{FS}, \mathrm{DM}, \mathrm{MMC}, \mathrm{CJO}$ and JHW recruited patients and collected data; SN and GG collected data and prepared the manuscript, KB, AJF and AA served on the DSMC; FG was leader of the core laboratory.

Competing interests None declared.

Patient consent Obtained.

Ethics approval Comité d'éthique du Centre hospitalier de l'Université de Montréal.

Provenance and peer review Not commissioned; externally peer reviewed.

Data sharing statement All available data can be obtained by contacting the corresponding author

(c) Article author(s) (or their employer(s) unless otherwise stated in the text of the article) 2017. All rights reserved. No commercial use is permitted unless otherwise expressly granted.

\section{REFERENCES}

1 Thompson BG, Brown RD, Amin-Hanjani S, et al. Guidelines for the management of patients with unruptured intracranial aneurysms: a guideline for Healthcare Professionals from the American Heart Association/American Stroke Association. Stroke 2015;46:2368-400.

2 Vernooij MW, Ikram MA, Tanghe HL, et al. Incidental findings on brain MRI in the general population. N Eng/ J Med 2007;357:1821-8.

3 Jalbert JJ, Isaacs AJ, Kamel H, et al. Clipping and coiling of unruptured intracranial aneurysms among Medicare beneficiaries, 2000 to 2010. Stroke 2015;46:2452-7.

4 Raymond J, Darsaut TE, Molyneux AJ. A trial on unruptured intracranial aneurysms (the TEAM trial): results, lessons from a failure and the necessity for clinical care trials. Trials 2011;12:64

5 Huang MC, Baaj AA, Downes $K$, et al. Paradoxical trends in the management of unruptured cerebral aneurysms in the United States: analysis of nationwide database over a 10-year period. Stroke 2011:42:1730-5.

6 Lai L, Morgan MK. The impact of changing intracranial aneurysm practice on the education of cerebrovascular neurosurgeons. J Clin Neurosci 2012;19:81-4.

7 Molyneux A, Kerr R, Stratton I, et al. International Subarachnoid Aneurysm trial (ISAT) of neurosurgical clipping versus endovascular coiling in 2143 patients with ruptured intracranial aneurysms: a randomised trial. Lancet 2002;360:1267-74.

8 Ferns SP, Sprengers ME, van Rooij WJ, et al. Coiling of intracranial aneurysms: a systematic review on initial occlusion and reopening and retreatment rates. Stroke 2009:40:e523-9.

9 Brown MA, Parish J, Guandique CF, et al. A long-term study of durability and risk factors for aneurysm recurrence after microsurgical clip ligation. J Neurosurg 2017;126:1-6. [Epub ahead of print].
10 Raymond J, Guilbert F, Weill A, et al. Long-term angiographic recurrences after selective endovascular treatment of aneurysms with detachable coils. Stroke 2003;34:1398-403.

11 Daou B, Chalouhi N, Starke RM, et al. Clipping of previously coiled cerebral aneurysms: efficacy, safety, and predictors in a cohort of 111 patients. J Neurosurg 2016;19:1-7. [Epub ahead of print].

12 Lad SP, Babu R, Rhee MS, et al. Long-term economic impact of coiling vs clipping for unruptured intracranial aneurysms. Neurosurgery 2013;72:1000-13.

13 Brinjikji W, Rabinstein AA, Lanzino G, et al. Patient outcomes are better for unruptured cerebral aneurysms treated at centers that preferentially treat with endovascular coiling: a study of the national inpatient sample 2001-2007. AJNR Am J Neuroradiol 2011:32:1065-70

14 Kotowski M, Naggara O, Darsaut TE, et al. Safety and occlusion rates of surgica treatment of unruptured intracranial aneurysms: a systematic review and metaanalysis of the literature from 1990 to 2011. J Neurol Neurosurg Psychiatry 2013;84:42-8.

15 Naggara ON, Lecler A, Oppenheim C, et al. Endovascular treatment of intracranial unruptured aneurysms: a systematic review of the literature on safety with emphasis on subgroup analyses. Radiology 2012;263:828-35.

16 Naggara ON, White PM, Guilbert F, et al. Endovascular treatment of intracranial unruptured aneurysms: systematic review and meta-analysis of the literature on safety and efficacy. Radiology 2010;256:887-97.

17 Raymond J, Darsaut TE, Kotowski M, et al. Unruptured intracranial aneurysms: why clinicians should not resort to epidemiologic studies to justify interventions. AJNR Am J Neuroradiol 2011;32:1568-9.

18 Raymond J. Managing unruptured aneurysms: the ethical solution to the dilemma. Can J Neurol Sci 2009:36:138-42.

19 Kotowski M, Naggara O, Darsaut TE, et al. Systematic reviews of the literature on clipping and coiling of unruptured intracranial aneurysms. Neurochirurgie 2012:58:125-31.

20 Wiebers DO, Whisnant JP, Huston J, et al. Unruptured intracranial aneurysms: natura history, clinical outcome, and risks of surgical and endovascular treatment. Lancet 2003;362:103-10

21 Brinjikji W, Rabinstein AA, Lanzino G, et al. Effect of age on outcomes of treatment of unruptured cerebral aneurysms: a study of the National Inpatient Sample 2001-2008. Stroke 2011;42:1320-4.

22 Brinjikji W, Rabinstein AA, Nasr DM, et al. Better outcomes with treatment by coiling relative to clipping of unruptured intracranial aneurysms in the United States, 20012008. AJNR Am J Neuroradiol 2011;32:1071-5.

23 Alshekhlee A, Mehta S, Edgell RC, et al. Hospital mortality and complications of electively clipped or coiled unruptured intracranial aneurysm. Stroke 2010;41:1471-6.

24 Raymond J, Guillemin F, Proust F, et al. Unruptured intracranial aneurysms. A critical review of the International Study of Unruptured intracranial aneurysms (ISUIA) and of Appropriate methods to address the clinical Problem. Interv Neuroradiol 2008:14:85-96.

25 Darsaut TE, Estrade L, Jamali S, et al. Uncertainty and agreement in the management of unruptured intracranial aneurysms. J Neurosurg 2014;120:618-23.

26 Darsaut TE, Findlay JM, Raymond J. The design of the Canadian UnRuptured Endovascular versus Surgery (CURES) trial. Can J Neurol Sci 2011:38:236-41.

27 Ergina PL, Cook JA, Blazeby JM, et al. Challenges in evaluating surgical innovation. Lancet 2009:374:1097-104.

28 McDougall CG, Johnston SC, Gholkar A, et al. Bioactive versus bare platinum coils in the treatment of intracranial aneurysms: the MAPS (Matrix and platinum science) trial. AJNR Am J Neuroradiol 2014:35:935-42.

29 Molyneux AJ, Clarke A, Sneade M, et al. Cerecyte coil trial: angiographic outcomes of a prospective randomized trial comparing endovascular coiling of cerebral aneurysms with either cerecyte or bare platinum coils. Stroke 2012;43:2544-50.

30 White PM, Lewis SC, Gholkar A, et al. Hydrogel-coated coils versus bare platinum coils for the endovascular treatment of intracranial aneurysms (HELPS): a randomised controlled trial. Lancet 2011;377:1655-62.

31 Raymond J, Klink R, Chagnon M, et al. Hydrogel versus Bare Platinum Coils in Patients with Large or Recurrent Aneurysms Prone to Recurrence after Endovascular Treatment: A Randomized Controlled Trial. AJNR Am I Neuroradio 2017:38:432-41.

32 Higashida RT, Lahue BJ, Torbey MT, et al. Treatment of unruptured intracranial aneurysms: a nationwide assessment of effectiveness. AJNR Am I Neuroradiol 2007;28:146-51.

33 Macdonald RL. Editorial: clip or coil? six years of follow-up in BRAT. I Neurosurg 2015;123:605-8

34 Darsaut TE, Kotowski M, Raymond J. How to choose clipping versus coiling in treating intracranial aneurysms. Neurochirurgie 2012;58:61-7.

35 The Cochrane Collaboration. Cochrane Handbook for Systematic Reviews of Interventions Version 5.1.0. www.cochrane-handbook.org.

36 Raymond J, Darsaut TE, Altman DG. Pragmatic trials can be designed as optimal medical care: principles and methods of care trials. J Clin Epidemiol 2014:67:1150-6. 


\section{Surgical clipping or endovascular coiling for unruptured intracranial aneurysms: a pragmatic randomised trial}

Tim E Darsaut, J Max Findlay, Elsa Magro, Marc Kotowski, Daniel Roy, Alain Weill, Michel W Bojanowski, Chiraz Chaalala, Daniela lancu, Howard Lesiuk, John Sinclair, Felix Scholtes, Didier Martin, Michael M Chow, Cian J O'Kelly, John H Wong, Ken Butcher, Allan J Fox, Adam S Arthur, Francois Guilbert, Lu Tian, Miguel Chagnon, Suzanne Nolet, Guylaine Gevry and Jean Raymond

J Neurol Neurosurg Psychiatry 2017 88: 663-668 originally published online June 20, 2017

doi: 10.1136/jnnp-2016-315433

Updated information and services can be found at:

http://jnnp.bmj.com/content/88/8/663

\section{These include:}

References This article cites 35 articles, 15 of which you can access for free at: http://jnnp.bmj.com/content/88/8/663\#BIBL

Email alerting

Receive free email alerts when new articles cite this article. Sign up in the service box at the top right corner of the online article.

\section{Notes}

To request permissions go to:

http://group.bmj.com/group/rights-licensing/permissions

To order reprints go to:

http://journals.bmj.com/cgi/reprintform

To subscribe to BMJ go to:

http://group.bmj.com/subscribe/ 